\title{
Development and Distinction of Rhizoma rodgersiae with HPLC-DAD Fingerprint Analysis Combined with Chemical Pattern Recognition Techniques
}

\author{
Yan Wang1, Yang Jin ${ }^{2 *}$ \\ ${ }^{1}$ College of Pharmacy and Chemistry, Dali University, Dali, China \\ ${ }^{2}$ Department of Pharmacy, Traditional Chinese Medicine Hospital of Dali, Dali, China \\ Email: *kingyoung520530@sina.com
}

How to cite this paper: Wang, Y. and Jin, Y. (2017) Development and Distinction of Rhizoma rodgersiae with HPLC-DAD Fingerprint Analysis Combined with Chemical Pattern Recognition Techniques. American Journal of Analytical Chemistry, 8, 681-689. https://doi.org/10.4236/ajac.2017.811050

Received: August 31, 2017

Accepted: November 3, 2017

Published: November 6, 2017

Copyright $\odot 2017$ by authors and Scientific Research Publishing Inc. This work is licensed under the Creative Commons Attribution International License (CC BY 4.0).

http://creativecommons.org/licenses/by/4.0/ (c) (i) Open Access

\begin{abstract}
In this paper, the feasibility and advantages of employing high performance liquid chromatographic-photodiode array detection (HPLC-DAD) fingerprint combined with chemical pattern recognition for quality consistency evaluation of widely used Rhizoma rodgersiae (RR) were investigated and demonstrated for the first time. The Similarity Evaluation System was employed to evaluate the similarities of 10 batches of RR sample; moreover, hierarchical clustering analysis (HCA) and principal component analysis (PCA) were also successfully applied to discriminate RR samples of different regions and seasons. Our results indicated that the seasonal variation had some influence on the chemical fingerprints of this herbal drug. This approach allowed the discrimination of RR samples from different sources. The current study demonstrated that fingerprint profiling coupled with chemical pattern recognition offered a reliable and efficient way to comprehensively assess the quality consistency of the tested samples.
\end{abstract}

\section{Keywords}

Chemical Pattern Recognition, HPLC-DAD Fingerprinting,

Quality Consistency Evaluation

\section{Introduction}

Rhizoma rodgersiae (RR), commonly known as Yan-Tuo in Chinese, is derived from the dried rhizome of Rodgersia sambucifolia hemsl in the family of Saxi- 
fragaceae [1]. It originated from China that mainly distributed in provinces of Yunnan, Guizhou, Sichuan, and Shanxi. Until now, RR has been extensively used as a traditional Chinese medicine (TCM) due to its explicit therapeutic effect for the treatment of bacillary dysentery, gastroenteritis and bronchitis [2]. Chemical investigations have demonstrated that polyphenols and saccharides were the main composition of this plant [3]; however, the quality control of the drug sample has only been evaluated by quantitative determination of bergenin, which was one of characteristic constituents in RR with pharmacological properties of cough-relieving, anti-HIV, antiarrhythmic and anti-inflammatory [4] [5] [6]. To date, studies with respect to the quality evaluation of the medicinal plant can rarely be found.

Chromatographic fingerprinting is believed to be a criterion for the quality assessment of TCM and herbal preparations by World Health Organization (WHO) and many authorities due to its capability of describing the holistic chemical ingredients of TCM, and it has been widely accepted and increasingly applied to quality control of herbal medicines [7] [8] [9] [10]. However, chromatographic fingerprint may contain numerous low intensity peaks; it is difficult to make a meaningful assessment by visual examination. Therefore, more effective classification methods, such as chemical pattern recognition, should be employed. Hierarchical clustering analysis (HCA), the unsupervised analysis, enables the visualization of the data in a reduced dimensional space built on the dissimilarities between samples with respect to their chemical composition [11] [12]. Sometimes, the results of HCA are confirmed by a third supervised analysis. This employs classification method, such as principal component analysis (PCA), allowing to separate samples of different sources [12] [13].

Considering the therapeutic importance of RR, in this present study, a feasible and reliable HPLC-DAD fingerprint analysis method for quality consistency evaluation of the medicinal plant collected from different regions and harvest times was developed for the first time, and then 10 batches of sample were analysed with the assistance of the "Similarity Evaluation System" software. Mathematical data handling techniques represented by HCA and PCA were also used to establish an objective pattern recognition system to discriminate samples from different sources.

\section{Materials and Methods}

\subsection{Chemicals and Materials}

Reference compounds (gallic acid and bergenin) were obtained from the National Institute for the Control of Pharmaceutical and Biological Products (NICPP, Beijing, China). Acetonitrile of HPLC grade was purchased from Tedia Company Inc. (Fairfield, USA). Phosphoric acid and other reagents were of analytical grade. Ten samples of RR (numbered 1 - 10) from different geographical locations between June 2007 and June 2010 were summarized in Table 1, and authenticated by Professor Deyuan Chen in Guiyang College of Chinese Medicine (Guiyang, China). 
Table 1. The information of 10 batches of Rhizoma rodgersiae samples in this study.

\begin{tabular}{cccc}
\hline Sample No. & Geographical regions & Harvest times & Similarity value \\
\hline 1 & Shuicheng, Guizhou, China & June 2007 & 0.998 \\
2 & Xingyi, Guizhou, China & July 2007 & 0.983 \\
3 & Weining, Guizhou, China & July 2007 & 0.992 \\
4 & Shuicheng, Guizhou, China & July 2008 & 0.995 \\
5 & Shuicheng, Guizhou, China & June 2010 & 0.983 \\
6 & Kunming, Yunnan, China & June 2007 & 0.958 \\
7 & Xuanwei, Yunnan, China & July 2008 & 0.996 \\
8 & Dali, Yunnan, China & June 2010 & 0.974 \\
9 & Jianchuan, Yunnan, China & June 2010 & 0.995 \\
10 & Heqing, Yunnan, China & June 2010 & 0.993 \\
\hline
\end{tabular}

\subsection{Instruments and Chromatographic Conditions}

Chromatographic fingerprinting was performed with a Shimadzu LC-20AD system (Tokyo, Japan), consisting of a photodiode array detector (SPD-M20A), low pressure quaternary pumps, online degasser and an autosampler. The chromatographic separation was carried out on an Alltima $\mathrm{C}_{18}$ column $(250 \mathrm{~mm} \times 4.6 \mathrm{~mm}$, $5 \mu \mathrm{m}$ ) (Alltech, USA) thermostated at $30^{\circ} \mathrm{C} \pm 0.15^{\circ} \mathrm{C}$. The mobile phase consisted of $0.1 \%(\mathrm{v} / \mathrm{v})$ phosphoric acid aqueous solution (Mobile phase A) and acetonitrile (Mobile phase B). Gradient elution was performed at a flow rate of $0.8 \mathrm{~mL} / \mathrm{min}$ using the following gradient program: $0.01-5 \mathrm{~min}, 1 \%-5 \% \mathrm{~B} ; 5-25 \mathrm{~min}, 5 \%$ $14 \% \mathrm{~B} ; 25-60 \mathrm{~min}, 14 \%-20 \% \mathrm{~B}$. The monitoring wavelength was set at $310 \mathrm{~nm}$. The injection volume was $10 \mu \mathrm{L}$.

\subsection{Sample and Standard Solution Preparations}

Appropriate amounts of reference standards were accurately weighed and transferred into $10-\mathrm{mL}$ volumetric flasks, and then dissolved with $60 \%(\mathrm{v} / \mathrm{v})$ methanol, which were used as the standard solutions. The roots of RR were powdered and then passed through a 40-mesh sieve. The dried powdered sample (1.00 g) was accurately weighed and extracted twice with $50 \mathrm{~mL} \mathrm{60 \%} \mathrm{(v/v)} \mathrm{methanol} \mathrm{for}$ one hour under reflux. The combined solution was evaporated using a rotary evaporator, and then diluted to $10 \mathrm{~mL}$ in a volumetric flask with $60 \%(\mathrm{v} / \mathrm{v})$ methanol. All samples and standard solutions were stored in a refrigerator at $4^{\circ} \mathrm{C}$ and warmed up to room temperature, and then filtered through $0.45 \mu \mathrm{m}$ membrane filters prior to HPLC analysis.

\subsection{Data Analysis}

The integrated chromatograms exported from CLASS-VP chromatography workstation were introduced into the professional software named Similarity Evaluation System for Chromatographic Fingerprint of Traditional Chinese Medicine (ver- 
sion 2004 A) for similarity analysis. The similarity values (correlative coefficients) of sample fingerprints versus the reference fingerprint were calculated automatically by the Similarity Evaluation System. Other data processing was performed using SPSS 19.0 (SPSS Inc., USA).

\section{Results and Discussions}

\subsection{Optimization of the Extraction and Chromatographic Conditions}

For the extraction solvents, six solutions $(30 \% \mathrm{EtOH}, 60 \% \mathrm{EtOH}, 95 \% \mathrm{EtOH}$, $30 \% \mathrm{MeOH}, 60 \% \mathrm{MeOH}$, and $100 \% \mathrm{MeOH}$ ) were evaluated, and other extraction conditions involved extraction method (ultrasonication and reflux), extraction repetitions (1, and 2) and extraction time (15, 30 and $60 \mathrm{~min}$ ) were also investigated. By comparing the sum numbers and responses of common peaks in each chromatogram at different conditions, the optimized conditions were selected and listed in detail in Section of 2.3.

For the HPLC conditions, different mobile phase systems (methanol-water, acetonitrile-water, methanol-phosphoric acid, acetonitrile-phosphoric acid, methanol-formic acid, acetonitrile-formic acid), chromatographic column (Alltima $\mathrm{C}_{18}$ column, $4.6 \times 250 \mathrm{~mm}, 5 \mu \mathrm{m}$; Diamosil $\mathrm{C}_{18}$ column, $4.6 \times 250 \mathrm{~mm}, 5 \mu \mathrm{m}$; Hypersil $\mathrm{ODS}_{2}$ column, $4.6 \times 250 \mathrm{~mm}, 5 \mu \mathrm{m}$ ), column temperature of 25,30 and $35^{\circ} \mathrm{C}$ and flow rate of $0.6,0.8$ and $1.0 \mathrm{~mL} / \mathrm{min}$ were tested. By comparing the resolution, baseline, sum numbers and responses of common peaks in each chromatogram at different conditions, the optimum analytical conditions were chosen and presented in detail in Section of 2.2.

In order to obtain a reliable detection wavelength, HPLC-DAD coupled with three dimensional plots (3D-plots) was used [13] [14]. With the satisfactory resolution, more peaks with high response were observed at $310 \mathrm{~nm}$; hence the monitoring wavelength was maintained at $310 \mathrm{~nm}$.

\subsection{Method Validation}

The retention time and chromatographic response of peak 4 were acceptable; it was hence selected as reference peak to calculate the relative retention time and relative peak area. The HPLC method for fingerprinting was validated in terms of precision, repeatability and sample stability. The precision was determined by injecting the same sample solution five times consecutively. The repeatability was examined by analyzing five replicates of the same batch of sample under the established extraction method. The sample stability was measured by testing a single sample solution stored at room temperature for $0,5,10,15$ and 24 hours. The validation results (Table 2) demonstrated that the relative standard deviations (RSDs) of relative retention time and relative peak area for common peaks in the precision, repeatability and sample stability tests were not beyond 5.0\%, respectively. Therefore, the HPLC method was acceptable and applicable for fingerprint analysis. (Sample No.2 was chosen at random for the method validation.) 


\subsection{HPLC Fingerprint and Similarity Analysis}

Under the optimised conditions, the HPLC fingerprints of 10 samples from various sources were obtained and presented in Figure 1. It was found that these

Table 2. The validation results of HPLC fingerprint analysis.

\begin{tabular}{|c|c|c|c|c|c|c|c|c|c|c|c|c|}
\hline \multirow{3}{*}{$\begin{array}{c}\text { Parameters } \\
\text { Peak Number }\end{array}$} & \multicolumn{4}{|c|}{ Precision } & \multicolumn{4}{|c|}{ Repeatability } & \multicolumn{4}{|c|}{ Stability } \\
\hline & \multicolumn{2}{|c|}{ RRT } & \multicolumn{2}{|c|}{ RPA } & \multicolumn{2}{|c|}{ RRT } & \multicolumn{2}{|c|}{ RPA } & \multicolumn{2}{|c|}{ RRT } & \multicolumn{2}{|c|}{ RPA } \\
\hline & Average & RSD & Average & RSD & Average & RSD & Average & RSD & Average & RSD & Average & RSD \\
\hline 1 & 0.605 & 0.131 & 0.397 & 3.703 & 0.604 & 0.110 & 0.356 & 3.169 & 0.605 & 0.133 & 0.382 & 3.308 \\
\hline 2 & 0.623 & 0.089 & 0.096 & 3.660 & 0.622 & 0.070 & 0.083 & 4.491 & 0.622 & 0.071 & 0.096 & 3.782 \\
\hline 3 & 0.801 & 0.061 & 0.584 & 0.530 & 0.801 & 0.037 & 0.584 & 4.028 & 0.801 & 0.041 & 0.586 & 0.944 \\
\hline 4 & 1.000 & 0.000 & 1.000 & 0.000 & 1.000 & 0.000 & 1.000 & 0.000 & 1.000 & 0.000 & 1.000 & 0.000 \\
\hline 5 & 1.113 & 0.063 & 27.933 & 0.407 & 1.113 & 0.064 & 28.041 & 2.025 & 1.114 & 0.052 & 28.048 & 0.389 \\
\hline 6 & 1.309 & 0.135 & 0.721 & 0.478 & 1.309 & 0.104 & 0.702 & 3.538 & 1.309 & 0.105 & 0.724 & 0.313 \\
\hline 8 & 1.781 & 0.171 & 0.347 & 2.562 & 1.781 & 0.157 & 0.333 & 3.958 & 1.781 & 0.144 & 0.348 & 2.736 \\
\hline 9 & 1.814 & 0.166 & 1.402 & 2.146 & 1.815 & 0.150 & 1.385 & 4.085 & 1.815 & 0.137 & 1.413 & 2.404 \\
\hline 10 & 2.036 & 0.190 & 0.604 & 2.198 & 2.037 & 0.183 & 0.576 & 2.852 & 2.037 & 0.162 & 0.599 & 1.814 \\
\hline 11 & 2.239 & 0.204 & 0.342 & 4.587 & 2.240 & 0.195 & 0.368 & 4.260 & 2.240 & 0.175 & 0.364 & 4.027 \\
\hline 12 & 2.272 & 0.203 & 0.291 & 2.040 & 2.273 & 0.189 & 0.291 & 4.794 & 2.273 & 0.171 & 0.292 & 2.373 \\
\hline 13 & 2.292 & 0.206 & 0.441 & 1.841 & 2.292 & 0.200 & 0.436 & 4.792 & 2.293 & 0.177 & 0.443 & 2.085 \\
\hline
\end{tabular}

RRT: relative retention time; RPA: relative peak area.

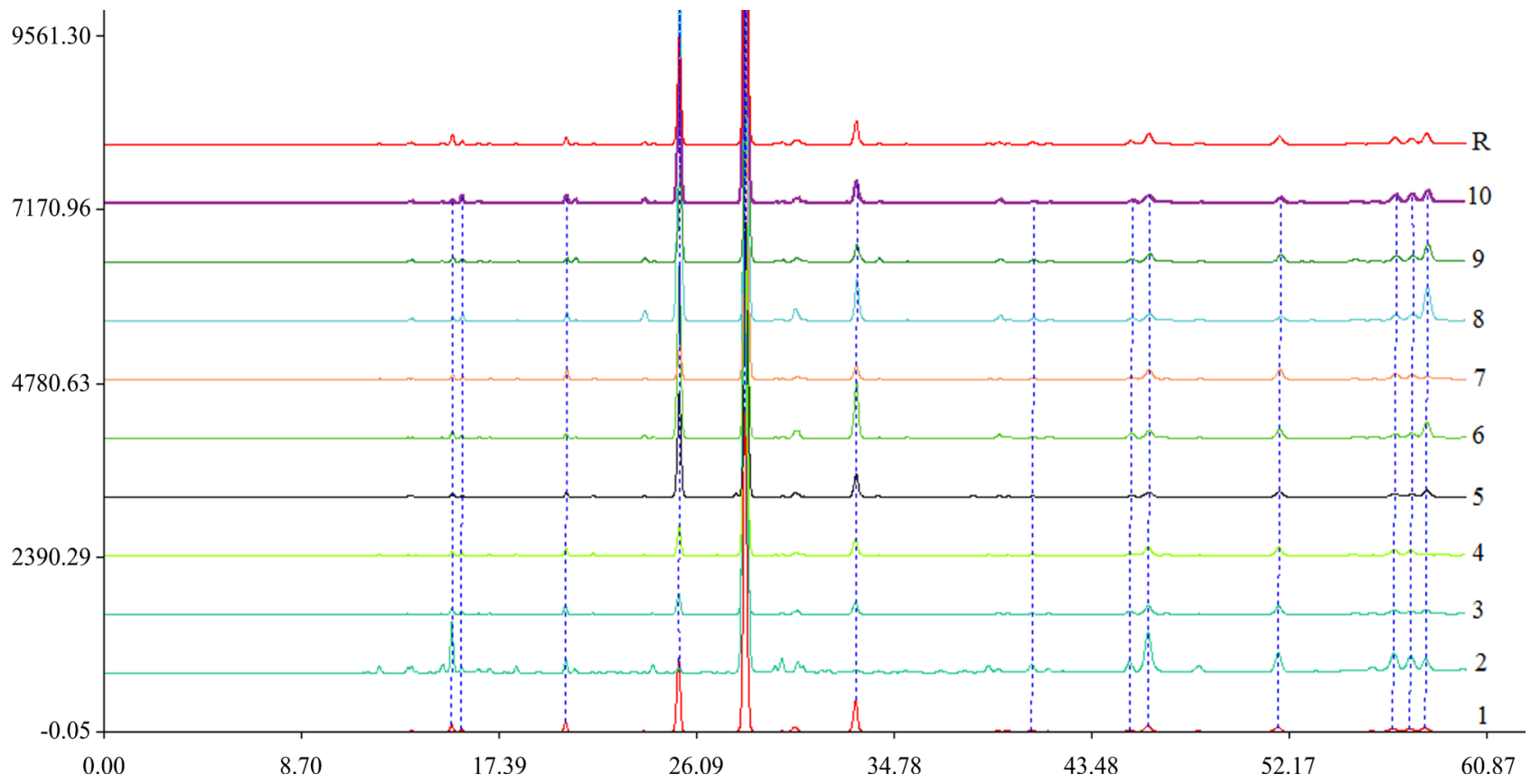

Figure 1. HPLC-DAD chromatographic fingerprints of 10 batches of Rhizoma rodgersiae samples from various sources at 310 $\mathrm{nm}$. Number is the number of sample in Table 1. 


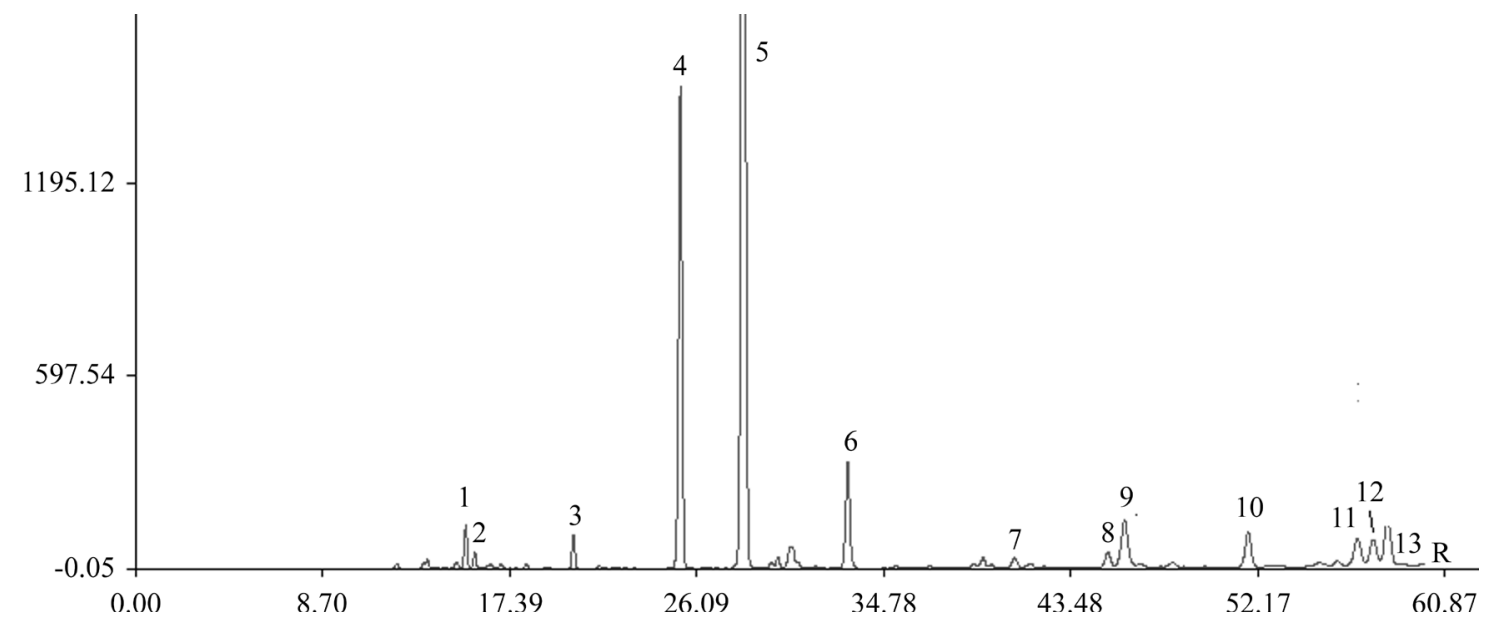

Figure 2. The reference fingerprint of the 10 tested samples.

samples had similar chemical profiles. Peaks existed in all chromatograms were regarded as common peaks, thus a total of 13 common peaks were found in the profiles, among which two (peaks 1 and 5) were unambiguous identified as gallic acid and bergenin based on comparison of UV spectra and retention times with the reference standards, and the reference fingerprint chromatogram was generated automatically by the similarity software and shown in Figure 2. From the correlative coefficients in Table 1, it could be seen that the similarity values of each chromatogram to the reference fingerprint chromatogram was found in the range of $0.958-0.998$. This indicated that similar quality properties were present in the herbal medicine samples regardless of either different geographical regions or seasonal differences, it was also suggested that similarity value was not sufficient enough for distinguishing samples of different sources.

\subsection{HCA Analysis}

For chromatographic fingerprint data matrix consisted of 10 rows (samples) and 13 columns (the relative peak areas of common peaks) was performed for HCA analysis based on the between-groups linkage method and Euclidean distance. The dendrogram shown in Figure 3 revealed two major clusters when the distance was set at 20. Four samples, which were collected in July (including No.2 4 and No.7), grouped as one distinct cluster (Cluster I), and the rest samples, which were collected in June clustered as Group II. The sample in the same cluster had more similar chemical fingerprints and internal qualities than others.

\subsection{PCA Analysis}

PCA, a statistical model that decomposes the data matrix into the low dimensions, was also applied to appraise the discriminating ability of the tested samples. The two-dimensional matrices $(10 \times 13)$ consisted of 10 objects and 13 variables, the objects were represented by the 10 batches of sample, and variables were represented by the standardized peak areas of fingerprint chromatogram. The score plot was shown in Figure 4, where the first component explained 43\% 


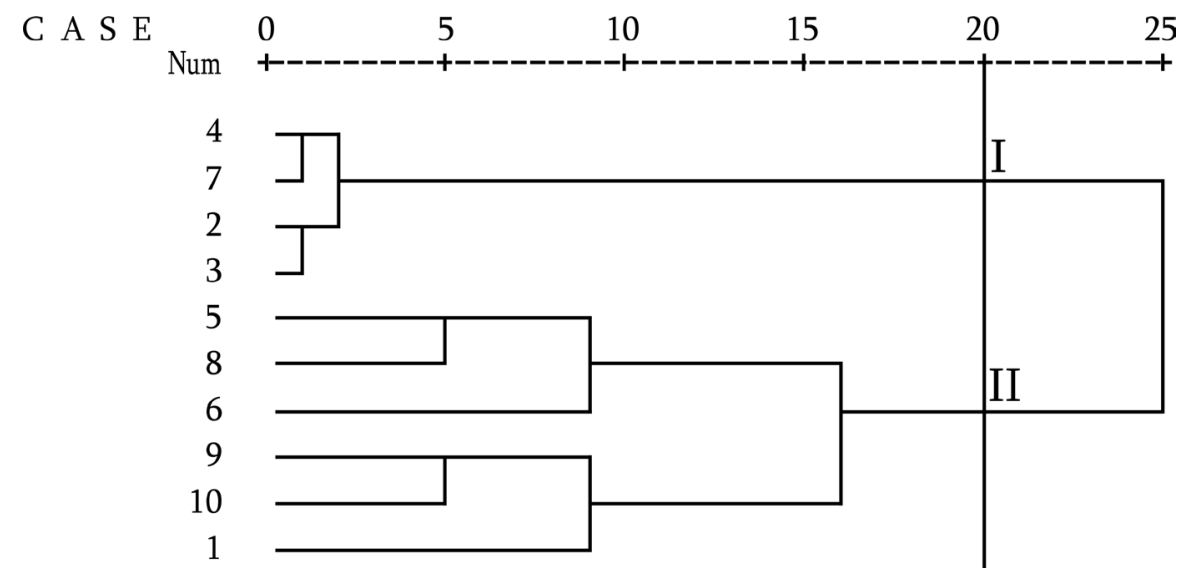

Figure 3. HCA dendrogram result for the chemical fingerprints of the 10 samples. Number is the number of sample in Table 1.

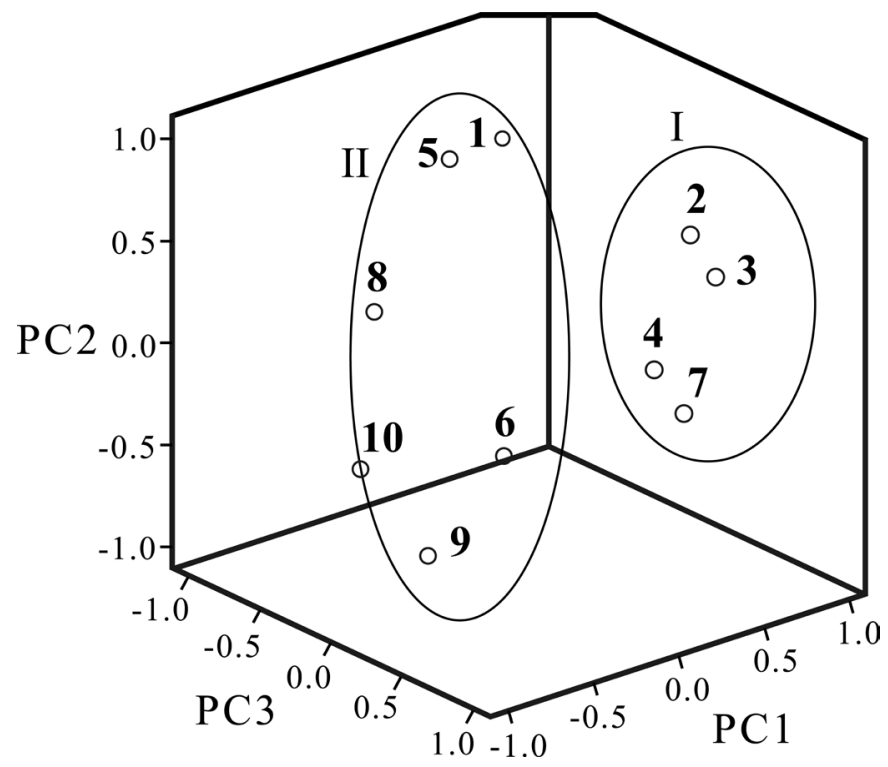

Figure 4. PCA score plot with 3PCs graph of the 10 samples. Number is the number of sample in Table 1.

of the variation, the second component $24 \%$, the third component $14 \%$ and the cumulative ratio exceeded $80 \%$. Based on the cumulative ratio $\geq 80 \%$ [13], the first three principal components (PC1, PC2 and PC3) were used to assess the consistency of the samples. 10 batches of samples were classified into two groups, which were marked I and II, respectively. In Group I, at the right of the score plot, contained No.2 - No.4 and No.7 samples. The Group II contained the rest of the tested samples, which were located on the left of the score plot. PCA plot displayed that a large distance separated the two clusters, especially the samples collected in June and the other samples collected in July, suggesting that seasonal variation may have some important influence on the internal quality of the medicinal material. The obtained results were in accordance with the HCA evaluation, and it was possible to deduce that the HCA and PCA implemented the classification of samples from seasonal differences. It was interesting that the herbal 
plant was not classified according to different regions; a possible explanation was that the provinces of Yunnan and Guizhou were vicinity in geographical location, and the natural growth environment of this wild plant was relatively similar. However, it should be noted that we do not have a sufficiently large data set in the current research, this also needs further research. In a next step, the chemical components which were responsible for the classification and provided a great potential to link to pharmacodynamic activities should be identified, isolated, and further examined.

\section{Conclusion}

In the present study, we used HPLC-DAD fingerprint combined with PCA and HCA to classify RR samples of different regions and seasons. The result demonstrated that the overall chemical profiles of all samples were consistent; however the contents of chemical components were varied among different origins. The reference fingerprint was used as the representative HPLC fingerprint to assess its chemical consistency from batch to batch. This is the first report for the batch to batch consistency assessment of RR and there are no data for quality analysis of RR with which to make a comparison. Using fingerprint technique combined with pattern recognition methods (HCA and PCA), the samples were successfully differentiated according to discrepant harvest times, indicating that seasonal variation may have some important influence on the chemical quality of the medicinal herbal. The established HPLC fingerprinting coupled with HCA and PCA analysis may provide some important reference for the development and improvement of the quality control of RR samples.

\section{Acknowledgements}

This research work was financially supported by the National Natural Science Foundation of China (Accession No. 81560695), and the Doctoral Research Fund of Dali University (Accession No. KYBS201512).

\section{Conflicts of Interest}

The authors declare no conflicts of interest.

\section{References}

[1] Weckerle, C.S., Ineichen, R., Huber, F.K. and Yang, Y.P. (2009) Mao's Heritage: Medicinal Plant Knowledge among the Bai in Shaxi, China, at a Crossroads between Distinct Local and Common Widespread Practice. Journal of Ethnopharmacology, 123, 213-228. https://doi.org/10.1016/j.jep.2009.03.014

[2] Deng, J.C., Xiao, X.H., Tong, X. and Li, G.K. (2010) Preparation of Bergenin from Ardisia Crenata Sims and Rodgersia Sambucifolia Hemsl Based on Microwave-Assisted Extraction/High-Speed Counter-Current Chromatography. Separation and Purification Technology, 74, 155-159. https://doi.org/10.1016/j.seppur.2010.05.018

[3] Hu, H.B., Zheng, S.Z., Zheng, X.D. and Shen, X.W. (2006) Chemical Constituents of Rodgersia sambucifolia Hemsl. ChemInform, 37. https://doi.org/10.1002/chin.200610214 
[4] Patel, D.K., Patel, K., Kumar, R., Gadewar, M. and Tahilyani, V. (2012) Pharmacological and Analytical Aspects of Bergenin: A Concise Report. Asian Pacific Journal of Tropical Disease, 2, 163-167. https://doi.org/10.1016/S2222-1808(12)60037-1

[5] Yu, W.S., Wang, Y.W., Zhang, Y.H., Zhang, D., Lan, J., Liu, Z.Y. and Gu, J.K. (2009) Quantitation of Bergenin in Human Plasma by Liquid Chromatography/Tandem Mass Spectrometry. Journal of Chromatography B, 877, 33-36. https://doi.org/10.1016/j.jchromb.2008.11.005

[6] Nazir, N., Koul, S., Qurishi, M.A., Najar, M.H. and Zargar, M.I. (2011) Evaluation of Antioxidant and Antimicrobial Activities of Bergenin and Its Derivatives Obtained by Chemoenzymatic Synthesis. European Journal of Medicinal Chemistry, 46, 2415-2420.

[7] Liu, Y., Zhao, Y.Y., Chen, H.B., Liang, H. and Zhang, Q.Y. (2013) Development of High-Performance Liquid Chromatographic Fingerprint for Quality Analysis of Hedysari Radix. Natural Product Research, 27, 1398-1403. https://doi.org/10.1080/14786419.2012.746341

[8] Kong, W.J., Wang, J.B., Zang, Q.C., Xing, X.Y., Zhao, Y.L., Liu, W., Jin, C., Li, Z.L. and Xiao, X.H. (2011) Fingerprint-Efficacy Study of Artificial Calculus bovis in Quality Control of Chinese Materia Medica. Food Chemistry, 127, 1342-1347.

[9] Lucio-Gutierrez, J.R., Garza-Juarez, A., Coello, J., Maspoch, S., Salazar-Cavazos, M.L., Salazar-Aranda, R. and Waksman de Torres, N. (2012) Multi-Wavelength HighPerformance Liquid Chromatographic Fingerprints and Chemometrics to Predict the Antioxidant Activity of Turnera diffusa as Part of Its Quality Control. Journal of Chromatography A, 1235, 68-76.

[10] Nguyen Hoai, N., Dejaegher, B., Tistaert, C., Nguyen Thi Hong, V., Riviere, C., Chataigne, G., Phan, V.K., Chau, V.M., Quetin-Leclercq, J. and Vander Heyden, Y. (2009) Development of HPLC Fingerprints for Mallotus Species Extracts and Evaluation of the Peaks Responsible for Their Antioxidant Activity. Journal of Pharmaceutical and Biomedical Analysis, 50, 753-763.

[11] Lu, X.F., Bi, K.S., Zhao, X. and Chen, X.H. (2012) Authentication and Distinction of Shenmai Injection with HPLC Fingerprint Analysis Assisted by Pattern Recognition Techniques. Journal of Pharmaceutical Analysis, 2, 327-333.

[12] Zhang, Y.T., Li, Q., Xing, H., Lu, X.F., Zhao, L.S., Qu, K.K. and Bi, K.S. (2013) Evaluation of Antioxidant Activity of Ten Compounds in Different Tea Samples by Means of an On-Line HPLC-DPPH Assay. Food Research International, 53, 847-856.

[13] Sun, G.X., Wu, Y., Liu, Z.B., Li, Y.F. and Guo, Y. (2014) Multiple Methods Were Combined to Monitor and Evaluate the Quality of TCM, and Make the Results More Reliable. Analytical Methods, 6, 838-849.

https://doi.org/10.1039/C3AY41670D

[14] Li, Y., Wu, T., Zhu, J.H., Wan, L.L., Yu, Q., Li, X.X., Cheng, Z.H. and Guo, C. (2010) Combinative Method using HPLC Fingerprint and Quantitative Analyses for Quality Consistency Evaluation of an Herbal Medicinal Preparation Produced by Different Manufacturers. Journal of Pharmaceutical and Biomedical Analysis, 52, 597-602. 